\title{
Causa rara de distensión abdominal aguda: apertura del conducto pancreático en un quiste hidatídico
}

\author{
A rare cause of acute abdominal distention: opening of the pancreatic duct into \\ hydatic cyst
}

\author{
Dr. Mehmet Agin ${ }^{a}$, Dr. Gokhan Tumgor ${ }^{a}$, Dra. Suzan Ícil ${ }^{b}$, Dr. Serdar İskit ${ }^{c}$, Dra. Derya Alabaz ${ }^{d} y$ \\ Dr. Huseyin T. Balle ${ }^{e}$
}

\begin{abstract}
RESUMEN
Introducción. El quiste hidatídico, causado por Echinococcus granulosus, se observa mayormente en el hígadoy los pulmones, aunque raramente también puede localizarse en cualquier órgano o tejido blando. En este artículo se presenta el caso de un paciente con quiste hidatídico pancreático con apertura del conducto pancreático en el quiste.

A propósito de un caso: Se atendió en nuestra clínica a un niño de 10 años de edad con distensión abdominal significativa y dolor en el epigastrio de 10 días de evolución. La concentración de amilasa sérica era de 3709 U / l y la inhibición de la hemaglutinación para hidatidosis era de 1/160. En la tomografía computarizada de abdomen se observaron dos lesiones separadas y ascitis, un quiste hidatídico de tipo CE2 en la región de la cola del páncreas y un quiste hidatídico de tipo CE1 en el lóbulo izquierdo del hígado. Se realizó el drenaje percutáneo del quiste ubicado en la cola del páncreas y se inició tratamiento con albendazol. Se retiró la sonda de drenaje, y desde ese momento se ha realizado el seguimiento del paciente sin que se observen complicaciones.

Palabras clave: quistehidatídico, páncreas, Echinococcus granulosus, pancreatitis, amilasa.
\end{abstract}

http:/ /dx.doi.org/10.5546/aap.2016.e346

\section{INTRODUCCIÓN}

La hidatidosis es una infección zoonótica de incidencia mundial, que afecta a los seres humanos y los animales, y es la causa de una pérdida económica significativa en los sectores

a. Departamento de Gastroenterología, Hepatología y Nutrición Pediátricas.

b. Departamento de Pediatría.

c. Departamento de Cirugía Pediátrica.

d. Departamento de Infectología Pediátrica.

e. Departamento de Radiología.

Facultad de Medicina de Çukurova Üniversitesi. Adana, Turquía.

Correspondencia:

Dr. Mehmet Agin: drmehmet47@yahoo.com

Financiamiento: Ninguno.

Conflicto de intereses: Ninguno que declarar.

Recibido: 23-12-2015

Aceptado: 25-2-2016 agrícolas y los sistemas de salud pública de las áreas endémicas. ${ }^{1} \mathrm{~A}$ menudo se observa parasitosis en los países con sectores agrícolas y ganaderos amplios y donde la medicina preventiva es deficiente. ${ }^{2,3}$ El quiste hidatídico, causado por Echinococcus granulosus, se observa mayormente en el hígado y los pulmones, aunque raramente también puede localizarse en cualquier órgano o tejido blando. En este artículo se presenta un paciente con quiste hidatídico pancreático con apertura del conducto pancreático en el quiste.

\section{A propósito de un caso}

Se atendió en nuestra clínica a un niño de 10 años de edad con dolor y distensión abdominal de 10 días de evolución, sin síntomas previos. La distensión había empeorado rápidamente, por lo que fue trasladado a nuestra clínica. En el examen físico se observó lo siguiente: peso de $24 \mathrm{~kg}$ (percentilo 10-25), altura de $137 \mathrm{~cm}$ (percentilo 90), temperatura corporal de $37^{\circ} \mathrm{C}$, frecuencia respiratoria de $24 / \mathrm{min}$, pulso de $88 / \mathrm{min}$ y presión arterial de 100/70 mm Hg. Debido a la distensión abdominal, el paciente tenía problemas respiratorios. Presentó dolor a la palpación del epigastrio y el abdomen. El resto de los sistemas y aparatos de órganos, y el hemograma completo eran normales. La concentración de amilasa sérica era de 3709 U / 1; la de amilasa en orina, 1641 U/1; y la de lipasa sérica, $323 \mathrm{U} / 1$; el resto de los parámetros bioquímicos eran normales (Tabla 1). En la ecografía abdominal se observó ascitis y una lesión quística lobulada en el cuadrante izquierdo del abdomen. En la tomografía computarizada (TC) abdominal se determinó la presencia de dos lesiones separadas y ascitis: un quiste hidatídico de tipo CE2 en la cola del páncreas $(14,5 \times 10 \mathrm{~cm})$, un quiste hidatídico de tipo CE1 en el lóbulo izquierdo del hígado (2 cm de diámetro) (Figura 1) y ascitis en las regiones perihepática y periesplénica (Figura 2). El valor de la prueba de inhibición de la hemaglutinación (IHA) para hidatidosis era de 1/160. La TC de cerebro y tórax realizada para investigar la presencia de otros quistes hidatídicos 
fue normal. Se realizó el drenaje percutáneo del quiste ubicado en la cola del páncreas y se inició tratamiento con albendazol. Una vez establecido el diagnóstico de hidatidosis pancreática, se inició la administración prequirúrgica de $15 \mathrm{mg} / \mathrm{kg}$ / día, que se continuó durante un mes después de la cirugía. Después del drenaje, la distensión abdominal mejoró. La concentración de amilasa en el líquido del drenaje era de 336502 U/1. Semejante concentración elevada indicaba que se había producido la apertura del conducto pancreático en el quiste hidatídico preexistente, lo que causó su pronto crecimiento. Con el drenaje percutáneo y el tratamiento con albendazol se observó una recuperación aparente en los resultados de laboratorio y clínicos. En la resonancia magnética (RM) de abdomen se observó que el tamaño del quiste pancreático se había reducido a $3,6 \mathrm{~cm}$ (Figura 3). Se retiró la sonda del drenaje y se trasladó al paciente a la clínica para su seguimiento. Actualmente ha transcurrido el primer año de seguimiento y no se han observado complicaciones.

TABLA 1: Hemograma y parámetros bioquímicos del paciente

\begin{tabular}{lccc}
\hline & Al momento del ingreso & Al momento del alta & Rango normal \\
\hline Leucocitos $\left(\mathrm{mm}^{3}\right)$ & 8700 & 5100 & $4500-13500$ \\
Hemoglobina $(\mathrm{g} / \mathrm{dl})$ & 12 & 11 & $11,5-15,5$ \\
Trombocitos $\left(\mathrm{mm}^{3}\right)$ & 603000 & 415000 & $150000-400000$ \\
Amilasa sérica $(\mathrm{U} / \mathrm{l})$ & 3709 & 120 & $30-100$ \\
Amilasa en orina (U/l) & 1641 & 416 & $400-600$ \\
Amilasa en el líquido del quiste (U/l) & 336502 & 125 & $30-100$ \\
Lipasa sérica (U/l) & 323 & 48 & $3-32$ \\
Proteínas totales (g/dl) & 5,4 & 5,6 & $6,4-8,1$ \\
Albúmina (g/dl) & 2,7 & 3,4 & $4-5,3$ \\
AST (U/l) & 87 & 44 & $5-45$ \\
ALT (U/l) & 81 & 37 & $5-45$ \\
GGT (U/l) & 8 & 8 & $5-32$ \\
Bilirrubina total (mg/dl) & 0,2 & 0,2 & $0,3-2$ \\
Bilirrubina directa (mg/dl) & 0,7 & 0,1 & $0,1-0,2$ \\
\hline
\end{tabular}

AST: aspartato aminotransferasa, ALT: alanina aminotransferasa, GGT: gamma glutamil transferasa.

FiguRA 1: Lesión quística de $145 \times 100 \mathrm{~mm}$ con membranas en la región posterior, ubicada en la cola del páncreas

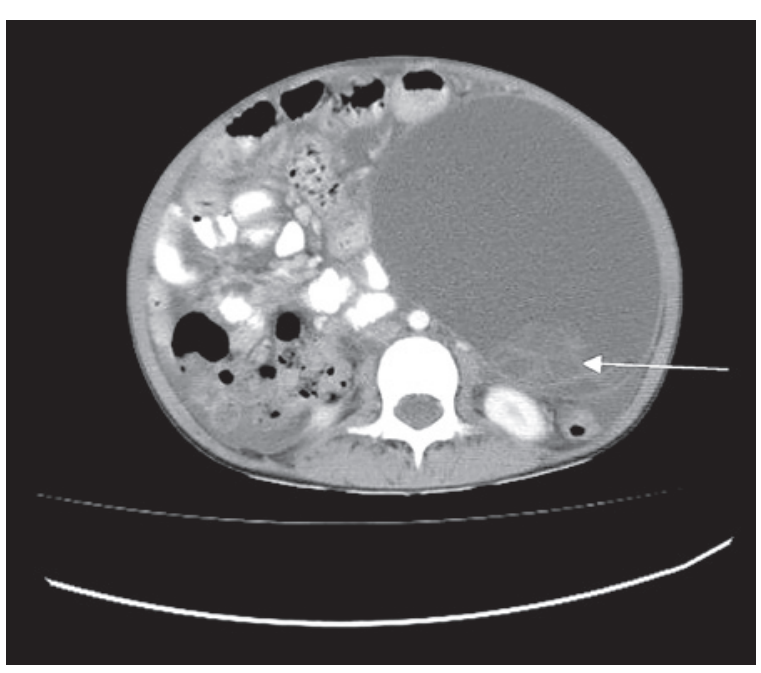

FigURA 2: Ascitis en las regiones perihepática y periesplénica

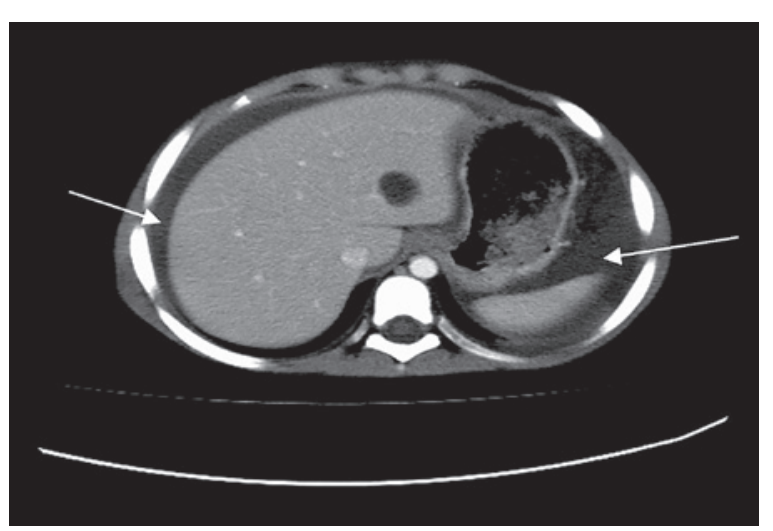




\section{DISCUSIÓN}

Según los informes, la cantidad anual de casos quirúrgicos de quiste hidatídico en Turquía es 0,9-6,6 cada 100000 personas; $^{4}$ sin embargo, en los estudios epidemiológicos se informaron tasas de prevalencia más elevadas, de 150-6884 cada 100000 personas. ${ }^{5,6} \mathrm{Si}$ bien el quiste hidatídico puede localizarse en cualquier órgano o tejido del organismo, los órganos más comúnmente afectados son el hígado (del $50 \%$ al $77 \%$ ), el pulmón (del 15\% al 47\%), el bazo (del 0,5\% al $8 \%$ ) y el riñón (del 2\% al 4\%). ${ }^{7,8}$ Aunque en los distintos estudios se han informado tasas de incidencia de quiste hidatídico pancreático variadas, las tasas son sistemáticamente inferiores al 1\%. El quiste hidatídico pancreático puede ser de tipo primario (que afecta exclusivamente al páncreas) o secundario (que afecta a varios órganos). ${ }^{9}$ Cuando afecta el páncreas, se extiende localmente a través de la invasión linfocítica de la región peripancreática, el sistema pancreático-biliar y la diseminación sanguínea. Se informó quiste pancreático en 54 pacientes; entre ellos, el quiste se ubicaba en la cabeza del páncreas en $21(38,8 \%)$, en la cola del páncreas en $18(33,3 \%)$, en el cuerpo y la cola del páncreas en $8(14,8 \%)$, en el cuerpo del páncreas en $5(9,2 \%)$, en la cabeza y el cuerpo en uno, y en el cuello en otro. ${ }^{10}$ Considerando que los quistes hidatídicos crecen con lentitud, la mayoría de los pacientes afectados no presenta síntomas durante años. Sin embargo, en los pacientes sintomáticos, los síntomas varían y dependen de la localización, el tamaño y la posición respecto de los órganos vecinos. ${ }^{11}$ Las complicaciones más graves de la

FIgURA 3: Lesión coincidente con quiste hidatídico de $39 x$ $36 \mathrm{~mm}$, que contiene la membrana germinativa, observada en la cola del páncreas en la secuencia axial ponderada en T2

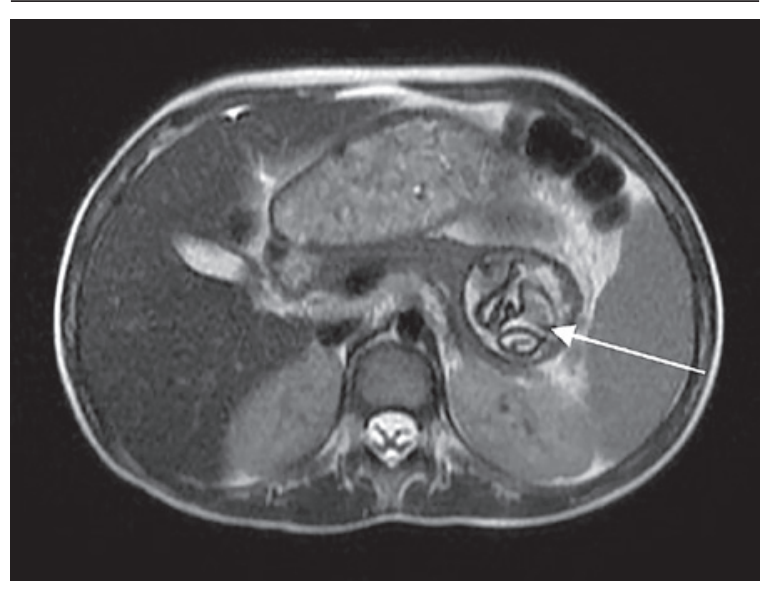

hidatidosis pancreática son la ictericia, la colangitis y la pancreatitis; todas estas complicaciones pueden aparecer como resultado de la fistulización del contenido del quiste hacia el conducto pancreático o las vías biliares o la compresión externa de los conductos por parte del quiste. ${ }^{11}$ Los pacientes sintomáticos suelen tener dolor abdominal, náuseas y vómitos.

Podrían presentar hepatitis, colangitis, pancreatitis, absceso pancreático y fístula pancreática, según la presión causada por el quiste sobre el conducto pancreático o la erosión del quiste hacia el conducto. ${ }^{12,13}$ El diagnóstico del quiste hidatídico pancreático es muy difícil dado que los síntomas y las manifestaciones son muy similares a los observados en otros tipos de quistes pancreáticos. Deben emplearse técnicas clínicas y de diagnóstico por imágenes radiológicas y análisis serológicos. Dado que los análisis serológicos son asequibles y fáciles de utilizar, se emplean en el diagnóstico y seguimiento de la enfermedad en países donde el quiste hidatídico es endémico. En los informes publicados se describen valores de sensibilidad diagnóstica para la IHA de entre el $51 \%$ y el $60,9 \% .{ }^{14,15}$

Puesto que no existe un protocolo estándar para el tratamiento del quiste hidatídico, el enfoque adoptado debería depender de los resultados de las pruebas clínicas, radiológicas y serológicas. ${ }^{2}$ El tratamiento del quiste hidatídico puede ser quirúrgico o clínico mediante el método de punción, aspiración, inyección y reaspiración (PAIR). Como la prueba de IHA está disponible en nuestro hospital y los resultados se obtienen con rapidez, la empleamos en nuestro paciente; los valores que se obtuvieron fueron significativamente elevados. La concentración de amilasa en suero, en orina y en el líquido del drenaje era alta. Debido a que nuestro paciente tenía un quiste pancreático muy grande y a que la concentración de amilasa en el líquido del drenaje era muy elevada, se consideró que el conducto pancreático tenía una apertura en el quiste. La ascitis no se consideró un hallazgo significativo, sino probablemente una pérdida mecánica pequeña debido a la gran presión ejercida por el quiste, el cual clínicamente podría haberse confundido con ascitis. Por lo tanto, es importante hacer una ecografía antes de decidir la realización de una punción abdominal, tal como se hizo en nuestro paciente para prevenir la diseminación del quiste hidatídico.

Si bien las concentraciones elevadas de amilasa y lipasa, la presencia de dolor abdominal y un 
quiste de gran tamaño en el páncreas sugieren inicialmente pancreatitis, el dolor abdominal se explica por la distensión del quiste y la presión de este sobre el abdomen. El aumento de la amilasa sérica podría estar relacionado con la secreción hacia el quiste (las membranas quísticas son semipermeables, especialmente al inicio, cuando no están calcificadas ni engrosadas).

Se inició tratamiento con albendazol y se utilizó el método PAIR. Durante el seguimiento, se observó que el tamaño del quiste se había reducido y que los parámetros clínicos y de laboratorio habían mejorado. Desde ese momento, el seguimiento del paciente ha sido ambulatorio, sin que se observen complicaciones.

A modo de conclusión, el quiste hidatídico, que es endémico en Turquía y la región, puede evolucionar de manera asintomática o generar síntomas y manifestaciones según su localización y el órgano afectado. Si bien el quiste hidatídico pancreático es raro, debe considerarse en primer lugar en el diagnóstico diferencial de los quistes pancreáticos, en especial en las regiones endémicas. Aquí describimos el caso de este paciente debido a sus complicaciones, la presencia de ascitis y dolor abdominal y epigástrico como consecuencia de un quiste hidatídico grande ubicado -raramente- en el páncreas, con apertura del conducto pancreático, y tratado de manera satisfactoria con PAIR y albendazol.

\section{REFERENCIAS}

1. Rojo-Vazquez FA, Pardo-Lledias J, Francos-Von Hunefeld M,Cordero-Sánchez M, et al.Cystic echinococcosis inSpain: current situation and relevance for other endemic areas in Europe. PLoS Negl Trop Dis 2011;5(1): e893.

2. Sayek I, Tirnaksiz MB, Dogan R. Cystic hydatid disease: current trends in diagnosis and management. Surg Today 2004;34(12):987-96.

3. Biava MF, Dao A, Fortier B. Laboratory diagnosis of cystic hydatic disease. Word J Surg 2001;25(1):10-4.

4. Altintas N. Past to present: echinococcosis in Turkey. Acta Trop 2003;85(2):105-12.

5. Ok UZ, Ozkol M, Kilimcio lu AA, Dinç G, et al. Provincebased study using sampling method to investigate the prevalence of cystic echinococcosis among primary school children in Manisa, Turkey. Acta Trop 2007;103(2):116-22.

6. Altintas N, Yazar S, Yolasigmaz A, Akisü C,et al. A seroepidemiological study of cystic echinococcosis in zmir and its surroundings area. Helminthologia 1999;36(1):19-23.

7. Eris C, Akbulut S, Yildiz MK, Abuoglu H, et al. Surgical approach to splenic hydatid cyst: single center experience. Int Surg 2013;98(4):346-53.

8. Yarlagadda P, Yenigalla BM, Penmethsa U, Myneni RB. Primary pancreatic echinococcosis. Trop Parasitol 2013;3(2):151-4.

9. Patil DS, Jadhav KV, Ahire PP, Patil SR, et al. Pancreatic hydatid presenting as an intercostal hernia. IJMAS 2013;2(3):255-8.

10. Akbulut S, Yavuz R, Sogutcu N, Kaya B, et al. Hydatid cyst of the pancreas: Report of an undiagnosed case of pancreatic hydatid cyst and brief literature review. World J Gastrointest Surg 2014;6(10):190-200.

11. Trigui A, Rejab H, Guirat A, Mizouni A,et al. Hydatid cyst of the pancreas About 12 cases. Ann Ital Chir 2013;84(2):165-70.

12. Wong S, Braghirolli-Neto O, Min Zu, Buckels J, Mirza D. Hydatid liver disease as a cause of recurrent pancreatic head. J R Coll Surg Edin 1999;44(6):407-9.

13. Barrera MC, Villanua J, Barrena JF, Nogues A. Pancreatic Hydatid disease. Pediatr Radiol 1995;25(Suppl 1):S169-70.

14. Gonlugur U, Ozcelik S, Gonlugur TE, Arici S, et al. The retrospective annual surgical incidence of cystic echinococcosis in Sivas, Turkey. Zoonoses Public Health 2009;56(5):209-14.

15. Wuestenberg J, Gruener B, Oeztuerk S, Mason RA, et al. Diagnostics in cystic echinococcosis: serology versus ultrasonography. Turk J Gastroenterol 2014;25(4):398-404. 